\title{
Investigation on Automatic Feeding Machine for Spring Supporting Pin Controlled by PLC
}

\author{
Bin Cai ${ }^{a}$, Guolin $\mathrm{Hu}^{\mathrm{b}}$ \\ Jiangxi Vocational College of Mechanical \& Electrical Technology, Nanchang, 330013, China. \\ a379190074@qq.com,b1025222495@qq.com
}

\begin{abstract}
This paper mainly studies the enterprise production based on the actual needs with spring support pin automatic feeding machine. This automatic feeding machine has been illustrated from the structure, working principle and characteristics, etc. This automatic feeding machine has many advantages such as high efficiency, low cost, and high quality etc.
\end{abstract}

Keywords: Structural Design; Spring Support Pin; Automatic Feeding Machine.

\section{Introduction}

In recent years, the booming spring manufacturing and supporting industry in China, but the current product line workers manual feeding prone to danger, and the problem of low efficiency, in order to improve spring support pin and related parts and components assembly speed, improve the production efficiency, save labor costs, in response to the "made in China 2025" plan of action, to need a spring support pin automatic feeding system to solve the above problems.

This project aims to develop a set of spring support pin based on PLC control automatic feeding system, the system can quickly identify spring and related parts, standard and non standard parts, used for spring support pin automatic feeding, after configuration tooling can satisfy the production three pin L series and B series models. The system consists of vibration disk, transitional storage mechanism, feeding mechanism, vacuum detection, vacuum suction, electric and pneumatic control system, etc. This system can meet the operation of indoor environment and can greatly meet the production load of small and micro enterprises.

\section{Research Status at Home and Abroad}

At present, the automatic feeding system has attracted the attention of researchers in China.Yang hongwei et al. [1] designed a set of automatic feeding mechanism for thin plate, which can take one piece out of a stack of neatly stacked thin steel plate without carrying a second piece.ChiHai [2] according to the China wafangdian bearing factory production line for tapered roller bearing cage renovation project requirements, design a set of tapered roller bearing cage automatic feeding mechanism, make its workers can replace manual feeding, to ensure the safety of the operating workers, and improve production efficiency.Feng Gui layers [3] by ProE movement simulation of the spring, such as automatic feeding machine simulation, make the original high-speed CNC spring sorting machine realizes the automatic feeding, improves the production efficiency, save the labor cost.Wei wenyi [4] analyzed and summarized the current situation and development of feed system of China's parallel twin screw extruder.Li yanrong et al. [5] studied and designed a set of automatic blanking and automatic outputting devices for stamping, and made the stamping system work orderly through program control.In summary, automatic feeding system has become a focus of domestic scholars, but there are few reports on the research of automatic feeding system for spring supporting pin.

Abroad, mainstream equipment has automatic feeding system to digital, networked, high-speed low consumption, more environmental protection and the direction of human development, as the spring manufacturing products to lighter weight, less material, more environmental protection and the development trend of visual, spring manufacturing in the developed countries have entered the industrial age 4.0, spring support pin, automatic feeding system is more and more extensive research and practice. 
To sum up, the current domestic automatic feeding system for single process more self-contained production equipment, low production efficiency, low accuracy, severe waste of material and labor force, and a series of problems, restricted the development of the spring manufacturing and related products, restricted the spring to the thorough transformation of the manufacturing scale, specialization and precision manufacturing and ascend.

\section{Market Forecast and Development Trend}

Spring supporting pin is a key part of various parts and components. It has the characteristics of "small commodity, large market" and is widely used in aerospace, automobile manufacturing and other related industries. The number of cars in China is increasing year by year, and the spring supporting pin has more and more broad market prospects. It is an urgent problem to develop a set of automatic feeding system such as spring supporting pin.

At present, spring support pin is generally formed by cold extrusion process [1]. Compared with traditional cutting processing method, it can reduce energy consumption and improve work efficiency [2].The existing automatic spring support pin feeding machine on the production line is prone to misinstall or overinstall springs due to some technical problems in the process [3].Therefore, high defect rate and high production cost make it difficult to cope with market competition [4].In view of the shortage of the above technology, this paper introduces a kind of structure design for spring supporting pin automatic feeding machine, which can realize fast, stable and reliable spring feeding.

\section{The General Scheme of Spring Support Pin Automatic Feeding Machine}

The overall scheme is shown in figure 1 . The vibration disk is installed at the lower side of the equipment.

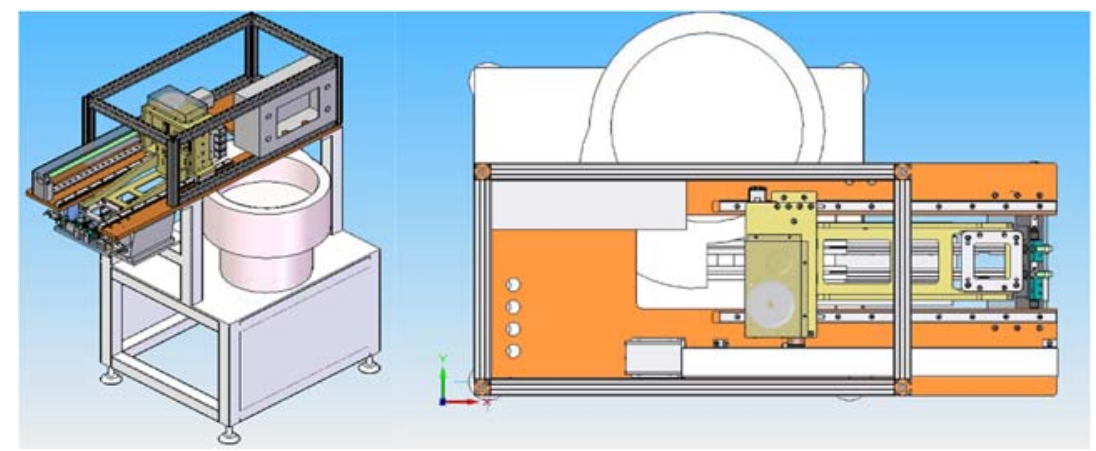

Fig 1.General scheme diagram of structure design of spring supporting pin automatic feeding machine

\section{PLC Control Flow Chart of Spring Supporting Pin Feeding Machine}

Spring support pin automatic feeding machine for electric control principle of the process, from the vibration plate and support pin the workpiece through the vacuum, the forwarding to the loading position, the chuck with vacuum pressure detector, ensure each time the vacuum suction cup, by conveying mechanism from the loading location by vacuum, once learned three support pin, accurate placement under welding equipment $(5.3 \mathrm{~mm})$ electrode hole $\Phi$ electrode position, in the sucker is expected at the same time equipped with vacuum pressure detector, ensure every time before the vacuum, feeding can detect whether learn three support pin, if the lack of support pin, at the right place to throw away has support pin, Refeed.The PLC control flow and wiring diagram of the spring support pin automatic feeding machine are shown in figure 2. 

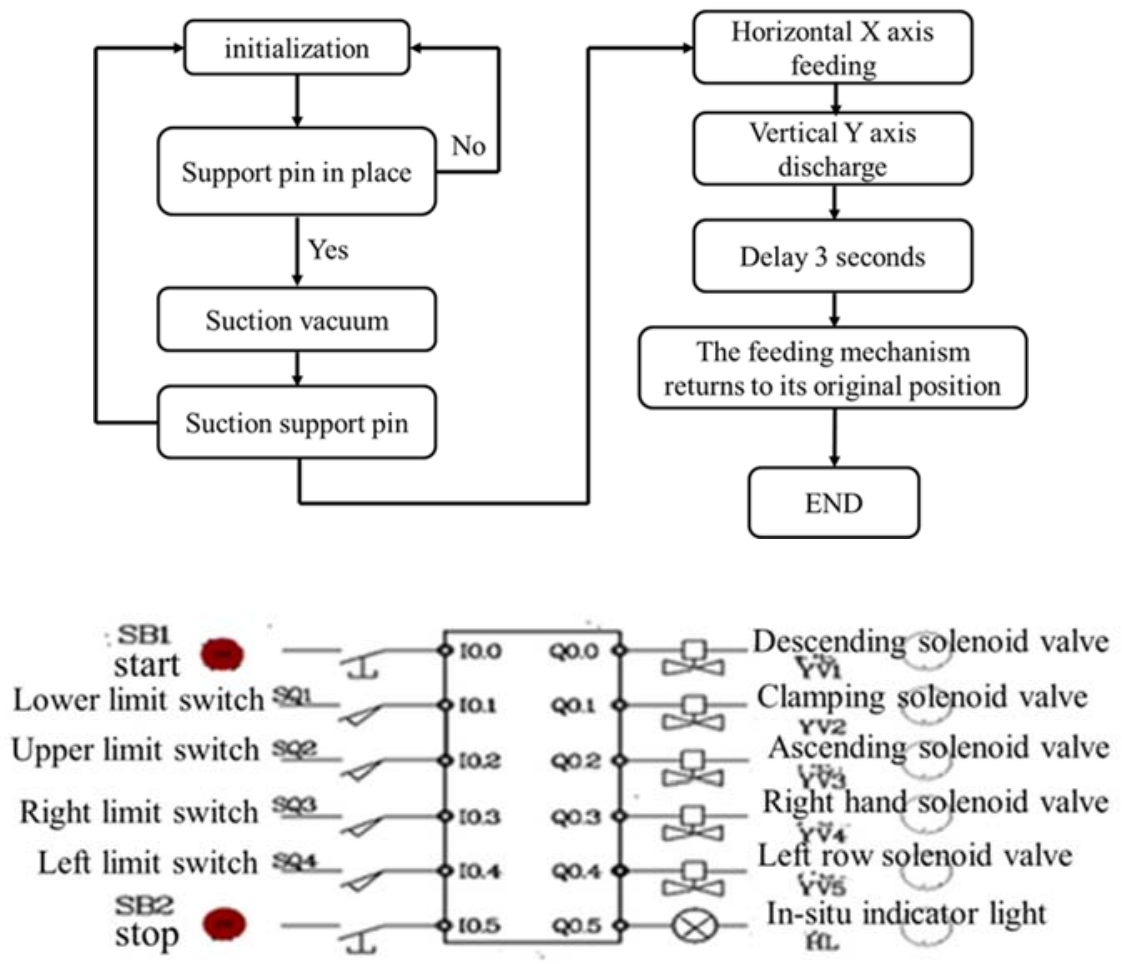

Fig 2. PLC control flow chart of spring supporting pin feeding machine

\section{Electrical Component}

\subsection{Electric Control Box}

Electrical control box should be sealed, install a low voltage electrical cabinet fluorescent lamp, make strong.Programmable controller (PLC) control system adopts Siemens product, you can use a portable monitor use computer programs to read and write operations and maintenance.Touch screen using Siemens brand.Programmable controller (PLC) I/O interface should have a certain allowance for later use.

The device sets the main operating box

1 equipment set up the main operating box, mainly produced in welding touch screen operation, the main operating box must be set aside at least 2 root of standby signal lines, the length of the signal lines should be appropriate to wear long, signal lines at both ends of the set of online code, easy to identify.All devices in the control box shall be marked, and all cables, signal lines and terminals shall be marked with cable code, which is of moderate size and not easy to fall off and fade away.No renewal of all cables from one terminal to another is allowed.The device layout of the main control box should be reasonable, so as to make it simple and clear. The modular structure should be used as far as possible to make the disassembly and installation simple and fast.

\subsection{The Power Supply Voltage}

The power supply voltage is $380 \mathrm{hz}++10 \%, 50 \mathrm{~Hz}$ (5-wire system) or single-phase $220 \mathrm{~V}, 50 \mathrm{~Hz}$ (3-wire system).Failure can be displayed (including failure indicator).Electrical, pneumatic and other systems have safety protection measures and devices such as accidental air break and power cut to maintain the correct position of working components. The equipment shall be connected with the welding machine, and the hardware and software related to the on-line demand shall be implemented by party b.

Pneumatic system

The pneumatic system consists of solenoid valve, cylinder, inductor, guide rail and low voltage electric appliance.Solenoid valve, cylinder USES SMC brand, inductor omron. The guideway USES the Taiwan HWIN brand.Zhengtai brand for low voltage electric appliance. 


\section{Performance Analysis}

Company has four production lines, each line configuration a spring support pin automatic feeding machine, about 60000 yuan or so, each device will save 1 workers per shift, save two workers a total of two class every day.If the minimum income of 40000 yuan a year per person, two workers salary is about 80000 yuan, and a spring support pin the application of the automatic feeding machine operation excluding maintenance fees, can recover the cost of a year.At the same time, also to increase the production efficiency to more than $15 \%$.

The global manufacturing industry will face difficulties labor costs rise, China's manufacturing has disappeared in the demographic dividend, the coming time of aging, improve the efficiency of manufacturing capacity and the reality of the demand, through the "industrial automation to replace artificial" became the trend of The Times.

\section{Acknowledgments}

This work was financially supported by the Jiangxi provincial department of education science research project Foundation (grant number GJJ161359).

\section{References}

[1]. Liu weiping, Wang mingquan. Development and application prospect of PLC[J]. Mechanical management development, 5(2009)78-80.

[2]. Zhang fenghua, Han baoling, Luo qingsheng, Shi lin, Huang lin. Design of a new industrial stacking robot control system based on PLC [J]. Computer measurement and control, 17(2009)2191-2196.

[3]. Jin lei, Wang jianyue. Application of configuration king and PLC in mechanical arm control system[J]. Chemical automation and instrumentation, 37(2010)103-106.

[4]. Chen yankui. The design method of PLC control system is briefly discussed[J]. China science and technology information, 20(2009)116-118. 